\title{
Singular perturbations of integral equations with degenerate kernels
}

Article in Integral Equations and Operator Theory · January 1996

DOI: $10.1007 / B F 01204605$

CITATION

1

1 author:
READS

16

\section{Carol Shubin}

California State University, Northridge

21 PUBLICATIONS 148 CITATIONS

SEE PROFILE

Some of the authors of this publication are also working on these related projects:

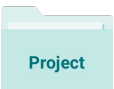

Project

Proceedings of the Conference in Honor of Leonid Pastur, Markov Processes and Related Fields, vol 9 \#4, (2003), 829-840. View project

American Mathematical Society, University Lecture Series, Vol 29 (Editors: I. Laba and C. Shubin), 2003. View project

All content following this page was uploaded by Carol Shubin on 26 May 2014. 


\title{
SINGULAR PERTURBATIONS OF INTEGRAL EQUATIONS WITH DEGENERATE KERNELS
}

\author{
CAROL ANN SHUBIN
}

Singularly perturbed Fredholm equations of the second kind are investigated. The kernels are allowed to have a jump discontinuity which vanishes at a point along the diagonal. Sufficient conditions for existence and uniqueness of solutions are found and the behavior of the solutions is studied.

\section{Introduction.}

This work is concerned with singularly perturbed integral equations on the interval $I=(a, b)$. Let $\epsilon$ be a small positive parameter. The perturbed equation is a Fredholm equation of the second kind

$$
\epsilon u_{\epsilon}(x)+\int_{a}^{b} K(x, y) u_{\epsilon}(y) d y=f(x)
$$

By setting $\epsilon=0$, we obtain the unperturbed equation

$$
\int_{a}^{b} K(x, y) u_{0}(y) d y=f(x)
$$

which is a integral equation of the first kind. We consider kernels $K(x, y)$ defined on $[a, b] \times[a, b]$ which have the form

$$
K(x, y)=\left\{\begin{array}{ll}
K_{1}(x, y) & y<x \\
K_{2}(x, y) & x<y
\end{array} .\right.
$$

We assume that $K_{1}(x, y)$ and $K_{2}(x, y)$ are smooth on the $[a, b] \times[a, b]$. The kernel $K(x, y)$ has a jump discontinuity along the diagonal

$$
K_{1}\left(x, x^{-}\right)-K_{2}\left(x, x^{+}\right)=a(x), \quad a(x) \in C^{\infty}[a, b]
$$


We study the degenerate case which occurs when $a(x)$ vanishes to order $k$ at one endpoint or at one interior point of the interval.

The connection between the solution to (1) and (2) in the nondegenerate case, when $a(x) \neq 0$ for $x \in[a, b]$, was considered in [LS] and [S]. This case is a subclass of elliptic singular perturbations of elliptic pseudodifferential equations. The regular degeneration of elliptic operators of higher order to elliptic operators was studied extensively by Višik and Ljusternik [VL]. An example of this situation is $\epsilon^{2} \Delta^{2}-\Delta$. Demidov [D], Eskin [E1], Frank $[F]$, Frank and Wendt [FW1,2,3], and Grubb [G] have studied elliptic pseudodifferential equations with a small parameter. When $a(x)$ vanishes at some point the unperturbed equation ceases to be elliptic and the methods of these works do not apply. Very little is known about the degenerate case but the behavior of the solutions appear to be far more complex than in the nondegenerate case. This work shows that the behavior of the solution to a Fredholm integral equation on an interval (1) which degenerates to an integral equation (2) (which is not a Fredholm equation) is already complicated. In particular the behavior of the solution depends on the sign of $a(x)$ in a neighborhood of the vanishing point. There is an analogy between the class of singularly perturbed integral equations considered here and the study of subelliptic pseudodifferential equations related to the oblique derivative problem, see [E3], [MP], [Sj], [T], and [VG]. These works consider the inversion of the first order differential operator, $L_{\xi}=\frac{d}{d x}+a(x)|\xi|$ where $|\xi|$ is a large parameter.

In $\S 1,2$, and 3 we take $a(x)=x^{k} b(x), k$ a positive integer, $b(x) \in C^{\infty}$, and $b(x)>0$. We show that (1) can be rewritten as

$$
L_{\epsilon}(B+\epsilon T) u_{\epsilon}(x)=f
$$

where $L_{\epsilon}$ is a first order differential operator

$$
L_{\epsilon}=\epsilon \frac{d}{d x}+a(x)
$$

$B$ is an associated integral operator of order -1 which does not depend on $\epsilon$ and $\epsilon T$ is a small perturbation of order -1. In particular, $B$ and $T$ map $L^{2}$ into $H^{1}$ and $B$ is assumed to have a one dimensional cokernel. Under this assumption, (1) is reduced to a regular perturbation problem. In our case, $B$ is shown to be the reduced operator; this is in contrast to the elliptic case when the reduced operator and the unperturbed operator coincide. Sufficient conditions are given for the existence of a unique solution to (1) in $L^{2}$ for any $f \in L^{2}$ and $0<\epsilon<\epsilon_{0}$.

$\S 4$ examines singularly perturbed integrals when $a(x)$ is replaced by $-a(x)$. We reduce the perturbed problem to regular perturbation problem by assuming that the reduced operator which maps $H^{-1}$ to $L^{2}$ has a one dimensional kernel. Sufficient conditions are given for the existence and uniqueness of the solution $u_{\epsilon}$ for any $f \in L^{2}$. 
At the end of sections 1,2 and 3 , the behavior of the solution $u_{\epsilon}$ as $\epsilon \rightarrow 0$ is described. In the nondegenerate case when $a(x) \neq 0$ on $[a, b], u_{\epsilon}$ tends to the solution of the unperturbed equation (2) in some Sobolev norm, see [E1]. This is not true in the degenerate case. The behavior of $u_{\epsilon}$ as $\epsilon \rightarrow 0$ is more complicated. Clearly, the solution to (2) is singular at $x=0$. Under suitable hypotheses, the solution of the perturbed equation converges to the solution of the reduced equation in $L^{2}[0,1]$ as $\epsilon$ tends to zero.

I would like to thank my advisor Professor G. Eskin for his valuable comments and interest in this work. Also I gratefully acknowledge NSF support DMS-9401735.

\section{Degeneracy at an endpoint.}

We consider equations (1) and (2) defined on [0,1]. The most general class of kernels for (2) which the results in this section bold is the following: let $K(x, y)=c(x) K_{0}(x, y)$ where $c(x)=x^{k} d(x), k \in Z^{+}, d(x)>0, d(x) \in C^{\infty}[0,1]$ and

$$
K_{0}(x, y)= \begin{cases}K_{01}(x, y) & x>y \\ K_{02}(x, y) & x<y\end{cases}
$$

with $K_{01}$ and $K_{02} \in C^{\infty}([0,1] \times[0,1])$ and $K_{0}\left(x, x^{-}\right)-K_{0}\left(x, x^{+}\right)=g(x)>0$. Let $b(x)=g(x) d(x)$ and $a(x)=x^{k} b(x)$. So we can write $K_{0}(x, y)=a(x) \Theta(x-y)+Q(x, y)$ where $\Theta(x)=1$ if $x>0$ and 0 otherwise. By Taylor's expansion about the diagonal

$$
Q(x, y)=\sum_{i=1}^{n} \alpha_{i}(x)(x-y)_{+}^{i}+\sum_{i=1}^{n} \beta_{i}(x)(x-y)_{-}^{i}+R_{n}(x, y)
$$

where $\alpha_{i}(x), \beta_{i}(x) \in C^{\infty}[0,1], i=1, \ldots, n, x_{+}^{i}=x^{i}$ if $x>0$ and 0 if $x<0, x_{-}^{i}=x^{i}$ if $x<0$ and 0 if $x>0$, and $R_{n}(x, y) \in C^{n}([0,1] \times[0,1])$.

Let us establish some notation which will be used throughout the paper. $c$ denotes a real positive number which may change from line to line. The $H^{1}-$ norm is defined as $\|v\|_{H^{1}} \leq\left\|\frac{d}{d x} v\right\|_{L^{2}}+c\|v\|_{L^{2}}$.

Thus the singularly perturbed integral equation (1) can be rewritten as

$$
\epsilon u_{\epsilon}(x)+a(x)\left(\int_{0}^{x} u_{\epsilon}(y) d y+\int_{0}^{1} Q(x, y) u_{\epsilon}(y) d y\right)=f(x)
$$

for $x \in(0,1)$ and $\epsilon$ is a small positive parameter. We will find conditions to ensure that there exists an unique solution to $(1.2), u_{\epsilon}(x) \in L^{2}(0,1)$, for each $f \in L^{2}(0,1)$. Then we will study the behavior of the solution $u_{\epsilon}$ as $\epsilon$ tends to zero.

We associate to (1.2) the first order differential operator

$$
L_{\epsilon}=\epsilon \frac{d}{d x}+a(x)
$$


The fundamental solution of the initial value problem for $L_{\epsilon}, x>0$, is given by

$$
G_{\epsilon}(x, y)=\frac{1}{\epsilon} \exp \left(-\int_{y}^{x} \frac{a(s)}{\epsilon} d s\right) \Theta(x-y) .
$$

Now we define the operator $R_{\epsilon}$ :

$$
R_{\epsilon} f(x)=\int_{0}^{1} G_{\epsilon}(x, y) f(y) d y \quad f \in L^{2}(0,1)
$$

and set

$$
E_{\epsilon}(x, y)=\exp \left(-\int_{y}^{x} \frac{a(s)}{\epsilon} d s\right) .
$$

Using integration by parts one can verify the relations:

$$
\begin{aligned}
R_{\epsilon} L_{\epsilon} w(x) & =w(x)-w(0) E_{\epsilon}(x, 0) & & w \in H^{1}[0,1] \\
L_{\epsilon} R_{\epsilon} g(x) & =g(x) & & g \in L^{2}(0,1) .
\end{aligned}
$$

By (1.3), $a=L_{\epsilon}-\epsilon \frac{d}{d x}$. So for $\frac{f}{a} \in H^{1}[0,1], R_{\epsilon} f$ can be written as $R_{\epsilon} f=R_{\epsilon} a\left(\frac{f}{a}\right)=$ $R_{\epsilon}\left(L_{\epsilon}-\epsilon \frac{d}{d x}\right) \frac{f}{a}$. Therefore, using (1.6a) we have

$$
R_{\epsilon} f=\frac{f}{a}-\frac{f}{a}(0) E_{\epsilon}(x, 0)-\epsilon R_{\epsilon} \frac{d}{d x} \frac{f}{a}
$$

We can rewrite (1.2) as

$$
L_{\epsilon} B u_{\epsilon}(x)-\epsilon T u_{\epsilon}(x)=f(x)
$$

where

$$
\begin{gathered}
B u_{\epsilon}(x)=\int_{0}^{x} u_{\epsilon}(y) d y+\int_{0}^{1} Q(x, y) u_{\epsilon}(y) d y \\
T u_{\epsilon}(x)=\int_{0}^{1} \frac{\partial Q}{\partial x}(x, y) u_{\epsilon}(y) d y
\end{gathered}
$$

Let's also define a regular perturbation of $B$ as follows:

$$
B_{\epsilon} v=B v-\epsilon R_{\epsilon} T v
$$

Now apply $R_{\epsilon}$ to (1.8) and use (1.6a) and (1.11) to obtain

$$
B_{\epsilon} u_{\epsilon}(x)=R_{\epsilon} f+c_{\epsilon} E_{\epsilon}(x, 0)
$$


where

$$
c_{\epsilon}=\int_{0}^{1} Q(0, y) u_{\epsilon}(y) d y .
$$

$c_{\epsilon}$ is an unspecified constant to be determined shortly.

We will modify the usage of term the 'reduced' equation in the following way. By (1.12) and (1.7), we have

$$
\begin{aligned}
B_{\epsilon} u_{\epsilon} & =R_{\epsilon} f+c_{\epsilon} E_{\epsilon}(x, 0) \\
& =\frac{f}{a}-\frac{f}{a}(0) E_{\epsilon}(x, 0)-\epsilon R_{\epsilon} \frac{d}{d x} \frac{f}{a}+c_{\epsilon} E_{\epsilon}(x, 0) .
\end{aligned}
$$

Then we are able to associate a 'reduced' equation to (1.2):

$$
B u_{0}=g \quad g \in H^{1}(0,1) .
$$

By (1.9), $B$ has index -1 as an operator from $L^{2}(0,1)$ into $H^{1}(0,1)$.

Lemma 1.1. Every element of the cokernel of $B$ has the form:

$$
v_{0}(x)=c_{1} \delta(x)+c_{2} \delta(x-1)+w(x)
$$

with $w(x) \in C^{\infty}[0,1]$.

Proof. First we show that if $u \in H^{-1}$ then $u=c \delta(x)+v$ where $v=\frac{d f}{d x}$, with $f \in L^{2}[0,1]$. To show this, let $u$ be a bounded linear functional on $H^{1}$. We will show that there exists a $c \in \mathbb{R}$ and $f \in L^{2}$ such that $u(h)=\operatorname{ch}(0)-\int_{0}^{1} h^{\prime} f d x$.

Let $H_{0}^{1}=\left\{h \in H^{1}: h(0)=0\right\}$. Define $T: H_{0}^{1} \rightarrow L^{2}$ such that $T(h)=h^{\prime}$. Note that $T$ is one-to-one and onto $L^{2}$. Now $u \circ T^{-1}$ is a linear functional on $L^{2}$ so by the Riesz Representation theorem there exists an $f$ such that $u\left(T^{-1} g\right)=-\int_{0}^{1} g f d x$. Let $g=T h$. Then $u(h)=-\int_{0}^{1} h^{\prime} f d x$. Let $c=u(1)$. Take any function $h \in H^{1}$. Then $h-h(0) \in H_{0}^{1}$ and since $u$ is linear, we have $u(h)=u(h-h(0))+u(h(0))=-\int_{0}^{1} h^{\prime} f d x+\operatorname{ch}(0)$.

Let $B^{*}$ be the adjoint of $B . B^{*}$ maps $H^{-1} \rightarrow L^{2}$. Next we show that if $u \in \operatorname{ker} B^{*}$, then $u=c \delta(x)+v$ where $v=\frac{d f}{d x}, f \in H^{k}$ for $k=0,1,2 \ldots$ The proof is by induction. By the above paragraphs, the case for $k=0$ holds by the definition of $f$. Assume true for $k-1$, $k \geq 1$. We will show it is true for $f \in H^{k}$.

Since $u \in \operatorname{ker} B^{*}$, then $u \in H^{-1}$ so $u=c \delta(x)+v$ where $v=\frac{d f}{d x}$ with $f \in L^{2}$. Then we have

$$
B^{*} u=\int_{x}^{1}\left(c \delta(y)+\frac{d f}{d y}\right) d y+\int_{0}^{1} Q(y, x)\left(c \delta(y)+\frac{d f}{d y}\right) d y=0 .
$$

Then

$$
\frac{d}{d x} B^{*} u=-\frac{d f}{d x}+\int_{0}^{1} \frac{\partial}{\partial x} Q(y, x) \frac{d f}{d y} d y+c \frac{d}{d x} Q(0, x)=0 .
$$


This implies that on $(0,1)$

$$
\frac{d f}{d x}=\int_{0}^{1} \frac{\partial}{\partial x} Q(y, x) \frac{d f}{d y} d y+c \frac{d}{d x} Q(0, x)
$$

$\operatorname{By}(1.1), Q(x, y)=\sum_{i=1}^{n} \alpha_{i}(x)(x-y)_{+}^{i}+\beta_{i}(x)(x-y)_{-}^{i}+R_{n}(x, y)$ with $\alpha_{i}(x), \beta_{i}(x) \in$ $C^{\infty}[0,1], i=1, . ., n$ and $R_{n}(x, y) \in C^{n}([0,1] \times[0,1])$. Therefore, $\frac{\partial}{\partial x} Q(y, x)$ has the expansion: $\frac{\partial Q}{\partial x}(y, x)=\rho_{0}(x) \Theta(x-y)+\gamma_{0}(x) \Theta(y-x)+$ smoother terms, with $\rho_{0}, \gamma_{0} \in C^{\infty}$. Also $\frac{\partial}{\partial x} Q(0, x) \in C^{\infty}[0,1]$. So if $f \in H^{k-1}$, then $\frac{d f}{d x} \in H^{k-2}$ and $\int_{0}^{1} \frac{\partial}{\partial x} Q(y, x) \frac{d f}{d y} d y \in$ $H^{k-1}$. Thus $\frac{d f}{d x} \in H^{k-1}$ on $(0,1)$, so $f \in H^{k}$ for all $k$. Consequently $f \in C^{\infty}[0,1]$. So we can conclude that $\frac{d f}{d x}=c_{1} \delta(x)+c_{2} \delta(x-1)+w$ where $w \in C^{\infty}[0,1]$, and then clearly $u$ has this form also.

We note that Lemma 1.1 is actually a special case of the transmission property - see [E2], chapter 23 .

We will make the following assumption, suggested by the fact that $B$ has index -1 .

Assumption 1.1 The cokernel of $B$ is one dimensional, and the constant $c_{1}$ of Lemma 1.1 is nonzero.

Let $($,$) denote the duality pairing between H^{1}(0,1)$ and its dual $H^{-1}(0,1)$. Then $\left(B u, v_{0}\right)=0$ for all $u \in L^{2}(0,1)$. Assumption 1.1 implies that there exists a bounded operator $R_{0}: H^{1}(0,1) \rightarrow L^{2}(0,1)$ such that

$$
\begin{array}{lc}
R_{0} B u=u & u \in L^{2}(0,1), \\
B R_{0} g=g & \text { if }\left(g, v_{0}\right)=0 \quad g \in H^{1}(0,1) .
\end{array}
$$

We apply $R_{0}$ to $(1.12)$ to obtain

$$
u_{\epsilon}(x)-\epsilon R_{0} R_{\epsilon} \int_{0}^{1} \frac{\partial Q}{\partial x}(x, y) u_{\epsilon}(y) d y=R_{0}\left(R_{\epsilon} f+c_{\epsilon} E_{\epsilon}(x, 0)\right) .
$$

Set

$$
T_{\epsilon} w(x)=\epsilon R_{0} R_{\epsilon} \int_{0}^{1} \frac{\partial Q}{\partial x}(x, y) w(y) d y, \quad w \in L^{2}(0,1)
$$

We will prove a series of lemmas which will show that $T_{\epsilon}$ is a bounded operator from $L^{2}(0,1)$ into $H^{1}(0,1)$ which satisfies the estimate

$$
\left\|T_{\epsilon} w\right\|_{L^{2}} \leq C \epsilon^{\frac{1}{2 k+2}}\|w\|_{H^{1}}
$$

These lemmas and a lemma describing the behavior of $c_{\varepsilon}$ as $\epsilon \rightarrow 0$ will imply the main results: 
Theorem 1.1. Suppose that Assumption 1.1 holds, then there exists an $\epsilon_{0}>0$ such that for $0<\epsilon<\epsilon_{0}$, there exists a unique solution $u_{\epsilon}(x) \in L^{2}(0,1)$, which is given by

$$
u_{\epsilon}(x)(x)=\left(I-T_{\epsilon}\right)^{-1} R_{0}\left(R_{\epsilon} f+c_{\epsilon} E_{\epsilon}(x, 0)\right)
$$

Theorem 1.2. Suppose Assumption 1.1 holds. If $\frac{f}{a} \in H^{1}[0,1]$ and $\frac{f}{a}$ is orthogonal to the cokernel of $B$, then $u_{\epsilon}(x) \rightarrow u_{0}(x)=R_{0}\left(\frac{\hat{L}}{a}\right)$ in $L^{2}[0,1]$ as $\epsilon \rightarrow 0$.

Lemma 1.2, The following estimates hold:

$$
\begin{gathered}
\left\|R_{0} E_{\epsilon}(x, 0)\right\|_{L^{2}} \leq c \epsilon^{-\frac{1}{2 k+2}} \\
\left\|E_{\epsilon}(x, 0)\right\|_{L^{2}} \leq c \epsilon^{\frac{1}{2 k+2}}
\end{gathered}
$$

Proof. Since $R_{0}: L^{2} \rightarrow H^{1}$ and $\left\|R_{0} E_{\epsilon}(x, 0)\right\|_{L^{2}}^{2} \approx\left\|\frac{d}{d x} E_{\epsilon}(x, 0)\right\|_{L^{2}}^{2}+\left\|E_{\epsilon}(x, 0)\right\|_{L^{2}}^{2}$, it suffices to consider $\left\|\frac{d}{d x} E_{\epsilon}(x, 0)\right\|_{L^{2}}^{2}$ and $\left\|E_{\epsilon}(x, 0)\right\|_{L^{2}}^{2}$. Using that $c_{1} x^{k}<a(x)<c_{2} x^{k}$ we have

$$
\begin{aligned}
\left\|\frac{d}{d x} E_{\epsilon}(x, 0)\right\|_{L^{2}}^{2} & =\int_{0}^{2} \frac{a(x)^{2}}{\epsilon^{2}} \exp \left(-\int_{0}^{x} \frac{2 a(s)}{\epsilon} d s\right) d x \\
& \approx \int_{0}^{1} \frac{x^{2 k}}{\epsilon^{2}} \exp \left(-c x^{k+1} / \epsilon\right) d x \\
& \approx \epsilon^{-\frac{1}{k+1}} \int_{0}^{\infty} t^{k / k+1} \exp (-c t) d t \\
& \approx c \epsilon^{-\frac{1}{k+1}}
\end{aligned}
$$

Hence (1.19).

Now

$$
\begin{aligned}
\left\|E_{\epsilon}(x, 0)\right\|_{L^{2}}^{2} & =\int_{0}^{1} \exp \left(-\int_{0}^{x} \frac{2 a(s)}{\epsilon} d s\right) d x \\
& \approx \int_{0}^{1} \exp \left(-c x^{k+1} / \epsilon\right) d x \\
& \approx \epsilon^{\frac{1}{k+1}} \int_{0}^{\infty} t^{k / k+1} \exp (-c t) d t \\
& \approx c \epsilon^{\frac{1}{k+1}}
\end{aligned}
$$

so (1.20) follows.

Lemma 1.3. If $f \in L^{2}$, then

$$
\left\|\epsilon R_{\epsilon} f\right\|_{L^{2}} \leq c \epsilon^{\frac{i}{k+1}}\|f\|_{L^{2}}
$$


Proof. Let $f \in L^{2}$. By Cauchy-Schwarz,

$$
\begin{aligned}
\left\|\epsilon R_{\epsilon} f\right\|^{2}= & \int_{0}^{1}\left(\int_{0}^{x} E_{\epsilon}(x, y) f(y) d y\right)^{2} d x \\
& \leq \int_{0}^{1} x \int_{0}^{x} E_{\epsilon}^{2}(x, y) f^{2}(y) d y d x .
\end{aligned}
$$

Interchanging the order of integration, we obtain

$$
\begin{aligned}
\left\|\epsilon R_{\epsilon} f\right\|^{2} \leq & \int_{0}^{1} \int_{y}^{1} x E_{\epsilon}^{2}(x, y) d x f^{2}(y) d y \\
& \leq \int_{0}^{1} \int_{y}^{1} x \exp \left(c \frac{y^{k+1}-x^{k+1}}{\epsilon}\right) d x f^{2}(y) d y
\end{aligned}
$$

By the change of variables $t=\frac{x^{k+1}-y^{k+1}}{\epsilon}$,

$$
\begin{aligned}
\left\|R_{\epsilon} f\right\|^{2} \leq & \int_{0}^{1} \int_{0}^{\infty}\left(\epsilon t+y^{k+1}\right)^{\frac{-k+1}{k+1}} \exp (-c t) d t f^{2}(y) d y \\
& \leq c \epsilon^{\frac{2 k}{k+1}}\|f\|^{2}
\end{aligned}
$$

Therefore, the lemma is proved.

Lemma 1.4. For $v \in H^{1}[0,1]$, then

$$
\left\|\epsilon \frac{d}{d x} R_{\epsilon} v\right\|_{L^{2}} \leq c \epsilon^{\frac{1}{2 k+2}}\|v\|_{H^{1}} .
$$

Proof. Let $v(x) \in H^{1}(0,1)$. Then

$$
\epsilon \frac{d}{d x} R_{\epsilon} v=v(x)-\frac{a(x)}{\epsilon} \int_{0}^{x} E_{\epsilon}(x, y) v(y) d y .
$$

Let

$$
I_{\epsilon}(x)=\frac{a(x)}{\epsilon} \int_{0}^{x} E_{\epsilon}(x, y) v(y) d y
$$

and

$$
I_{\epsilon}(x)=I_{\epsilon}^{1}(x)+I_{\epsilon}^{2}(x)
$$

where

$$
\begin{aligned}
& I_{\epsilon}^{1}(x)=\int_{0}^{x} \frac{a(y)}{\epsilon} E_{\epsilon}(x, y) v(y) d y \\
& I_{\epsilon}^{2}(x)=\int_{0}^{x} \frac{a(x)-a(y)}{\epsilon} E_{\epsilon}(x, y) v(y) d y .
\end{aligned}
$$


Clearly,

$$
I_{\epsilon}^{\dot{1}}(x)=v(x)-v(0) E_{\epsilon}(x, 0)-\int_{0}^{x} E_{\epsilon}(x, y) v^{\prime}(y) d y,
$$

By Lemma 1.3, $\left\|\epsilon R_{\epsilon} v^{\prime}\right\|_{L^{2}} \leq c \epsilon^{1 / k+1}\left\|v^{\prime}\right\|_{L^{2}}$. By Lemma 1.2, $\left\|E_{\epsilon}(x, 0)\right\|_{L^{2}} \leq c \epsilon^{1 / 2 k+2}$. So $\left\|\epsilon \frac{d}{d x} R_{\epsilon} v\right\|=\left\|v-I_{\epsilon}^{1}-I_{\epsilon}^{2}\right\| \leq c \epsilon^{1 / 2 k+2}\|v\|_{H^{1}}+\left\|I_{\epsilon}^{2}\right\|$.

We will use the observation that there exists some $c>0$ such that $|a(x)-a(y)| \leq$ $c\left|x^{k}-y^{k}\right|$ when estimating the $L^{2}(0,1)$ norm of $I_{\epsilon}^{2}(x)$.

$$
\begin{aligned}
I_{\epsilon}^{2}(x)= & \int_{0}^{x} \frac{a(x)-a(y)}{\epsilon} E_{\epsilon}(x, y) v(y) d y \\
& \leq c\|v\|_{L^{\infty}(0,1)} \int_{0}^{x} \frac{x^{k}-y^{k}}{\epsilon} \exp \left(c \frac{y^{k+1}-x^{k+1}}{\epsilon}\right) d y .
\end{aligned}
$$

Of course $\|v\|_{L^{\infty}} \leq c\|v\|_{H^{1}}<c$. By the change of variables $s=\frac{x^{k}-y^{k}}{\epsilon}$

$$
I_{\epsilon}^{2}(x) \leq c \epsilon \int_{0}^{\frac{x^{k}}{\epsilon}} s \exp (-c s x)\left(x^{k}-\epsilon s\right)^{\frac{1}{k}-1} d s .
$$

We write $I_{\epsilon}^{2}(x)=I_{\epsilon}^{3}(x)+I_{\epsilon}^{4}(x)$ where

$$
\begin{aligned}
& I_{\epsilon}^{3}(x)=\epsilon \int_{0}^{\frac{x^{k}}{2 \epsilon}} s \exp (-c s x)\left(x^{k}-\epsilon s\right)^{\frac{1}{k}-1} d s \\
& I_{\epsilon}^{4}(x)=\epsilon \int_{\frac{x^{k}}{2 \epsilon}}^{\frac{x^{k}}{\epsilon}} s \exp (-c s x)\left(x^{k}-\epsilon s\right)^{\frac{k}{k}-1} d s .
\end{aligned}
$$

Let us show

$$
\left\|I_{\epsilon}^{3}(x)\right\| \leq c \epsilon^{1 / 2 k+2}
$$

Using the fact that $\left(x^{k}-\epsilon s\right)^{\frac{1}{k}-1}$ is monotonically increasing for $0<s<\frac{x^{k}}{2 \epsilon}$ and integrating by parts, we find:

$$
I_{\epsilon}^{3}(x) \leq c_{1} x^{k+1} \exp \left(-\frac{c x^{k+1}}{\epsilon}\right)+c_{2} \epsilon\left(\exp \left(-\frac{c x^{k+1}}{2 \epsilon}\right)-1\right)
$$

By $(1.20)$, the $L^{2}(0,1)$ norm of the first term on the righthand side is less than $c \epsilon^{1 / 2 k+2}$. Clearly, the second term on the righthand side is $O(\epsilon)$. Finally, we consider $I_{\varepsilon}^{4}(x)$. We have:

$$
I_{\epsilon}^{4}(x) \leq c \frac{x^{k+1}}{\epsilon} \exp \left(-\frac{c x^{k+1}}{2 \epsilon}\right)
$$

Therefore,

$$
\left\|I_{\epsilon}^{4}(x)\right\| \leq c \epsilon^{1 / 2 k+2}
$$

So the lemma is proved. 
Lemma 1.5. $T_{\epsilon}$ is a bounded operator from $L^{2}[0,1] \rightarrow H^{1}[0,1]$ which satisfies the estimate

$$
\left\|T_{\epsilon} w\right\|_{L^{2}} \leq c \epsilon^{1 / 2 k+2}\|w\|_{H^{1}}
$$

Proof. By definition $T_{\epsilon} w=\epsilon R_{0} R_{\epsilon} \int_{0}^{1} \frac{\partial}{\partial x} Q(x, y) w(y) d y$. Let $v=\int_{0}^{1} \frac{\partial}{\partial x} Q(x, y) w(y) d y$ then $v \in H^{1}[0,1]$. Using Lemma 1.4, we have $\left\|\epsilon R_{0} R_{\epsilon} v\right\|_{L^{2}} \leq c \epsilon^{1 / 2 k+2}\|v\|_{H^{1}}$, So the result follows.

Note that for $R_{0}$ given by (1.16), we have

$$
R_{0} B_{\epsilon}=R_{0} B-\epsilon R_{0} R_{\epsilon} T=I-T_{\epsilon}
$$

By Lemma 1.5, for $\epsilon$ sufficiently small $I-T_{\epsilon}$ is invertible in $L^{2}(0,1)$, and

$$
\left(I-T_{\epsilon}\right)^{-1} R_{0} B_{\epsilon}=I
$$

Assumption 1.1 implies that $B_{\epsilon}$ has a one dimensional cokernel, call it $v_{\epsilon}(x)$, i.e.

$$
\left(B_{\epsilon} \imath t, v_{\epsilon}\right)=0 \text { for all } u \in L^{2}(0,1)
$$

Now we determine $v_{\epsilon}(x)$ in terms of $v_{0}(x)$. Let $B_{\epsilon}^{*}$ denote the adjoint of $B_{\epsilon}$, given by (1.11). We have

$$
\begin{aligned}
& B_{\epsilon}^{*}: H^{-1}(0,1) \rightarrow L^{2}(0,1) \\
& B_{\epsilon}^{*}=B^{*}-\epsilon\left(R_{\epsilon} T\right)^{*},
\end{aligned}
$$

and by the definition of $v_{\epsilon}(x)$,

$$
B_{\epsilon}^{*} v_{\epsilon}(x)=0 .
$$

Let $R_{0}^{*}$ denote the adjoint of $R_{0}$, defined by (1.9), which has the following properties:

$$
\begin{aligned}
& R_{0}^{*}: L^{2}(0,1) \rightarrow H^{1}(0,1) \\
& R_{0} B=I_{L^{2}(0,1)} \\
& \left(R_{0} B\right)^{*}=I_{L^{2}(0,1)}
\end{aligned}
$$

We will find an $h_{\epsilon} \in L^{2}(0,1)$ such that

$$
v_{\epsilon}(x)=v_{0}(x)+R_{0}^{*} h_{\epsilon}
$$

Let $T_{\epsilon}^{*}$ be the adjoint of $T_{\epsilon}$, i.e.

$$
T_{\epsilon}^{*}=\epsilon\left(R_{0} R_{\epsilon} T\right)^{*}
$$


By Lemma 1.5,

$$
\left\|T_{\epsilon}^{*} g\right\| \leq c \epsilon^{\frac{1}{2 k+2}}\|g\|
$$

so for $\epsilon$ sufficiently small $\left(I-T_{\epsilon}^{*}\right)^{-1}$ exists. Now we compute $h_{\epsilon}$ :

$$
\begin{aligned}
0=B_{\epsilon}^{*} v_{\epsilon}(x) & =\left(B^{*}-\epsilon\left(R_{\epsilon} T\right)^{*}\right)\left(v_{0}(x)+R_{0}^{*} h_{\epsilon}\right) \\
& =\left(I-T_{\epsilon}^{*}\right) h_{\epsilon}-\epsilon\left(R_{\epsilon} T\right)^{*} v_{0}(x) .
\end{aligned}
$$

Thus, we finally obtain

$$
h_{\epsilon}=\left(I-T_{\epsilon}^{*}\right)^{-1} \epsilon\left(R_{\epsilon} T\right)^{*} v_{0}(x) \in L^{2}(0,1) .
$$

Note that $v_{\epsilon}(x)$ is a small perturbation of $v_{0}(x)$.

Now we come to the crucial definition. Set

$$
c_{\epsilon}=-\frac{\left(R_{\epsilon} f, v_{\epsilon}\right)}{\left(E_{\epsilon}(x, 0), v_{\epsilon}\right)}
$$

provided that the denominator does not vanish. Note that since $R_{\epsilon} f=\frac{f}{a}-\frac{f}{a}(0) E_{\epsilon}(x, 0)-$ $\epsilon R_{\epsilon} \frac{d}{d x} \frac{f}{a}$ then $c_{\epsilon}=\frac{f}{a}(0)-\left(\frac{f}{a}, v_{\epsilon}(x)\right) /\left(E_{\epsilon}(x, 0), v_{\epsilon}(x)\right)+\left(\epsilon R_{\epsilon} \frac{d}{d x} \frac{f}{a}, v_{\epsilon}(x)\right) /\left(E_{\epsilon}(x, 0), v_{\epsilon}(x)\right)$.

Lemma 1.6. When Assumption 1.1 holds, $c_{\epsilon}$ given by (1.24), is well defined and $c_{\epsilon}=$ $-\frac{\left(\frac{f}{a}, v_{0}\right)}{c_{1}}+\frac{f}{a}(0)+O\left(\epsilon^{1 / k+1}\right)$

Proof. First we examine the denominator of $c_{\epsilon}(1.24)$ :

$$
\begin{aligned}
\left(E_{\epsilon}(x, 0), v_{\epsilon}\right) & =\left(E_{\epsilon}(x, 0), v_{0}+R_{0}^{*} h_{\epsilon}\right) \\
& =c_{1}+c_{2} E_{\epsilon}(1,0)+\left(E_{\epsilon}(x, 0), w\right)+\left(E_{\epsilon}(x, 0), R_{0}^{*} h_{\epsilon}\right) .
\end{aligned}
$$

The first term is nonzero by Assumption 1.1. The second term is exponentially small. The third term is $O\left(\epsilon^{1 / k+1}\right)$ by Cauchy-Schwarz and Lemma 1.2. Now we show that $\left|\left(E_{\epsilon}(x, 0), R_{0}^{*} h_{\epsilon}\right)\right| \leq c \epsilon^{3 / 2 k+2}$.

$$
\begin{aligned}
\left|\left(E_{\epsilon}(x, 0), R_{0}^{*} h_{\epsilon}\right)\right| & \leq\left\|R_{0} E_{\epsilon}(x, 0)\right\|_{L^{2}}\left\|h_{\epsilon}\right\|_{L^{2}} \\
& \leq c \epsilon^{-\frac{1}{2 k+2}}\left\|\left(1-T_{\epsilon}^{*}\right)^{-1} \epsilon\left(R_{\epsilon} T\right)^{*} v_{0}\right\|_{L^{2}} \\
& \leq c \epsilon^{-\frac{1}{2 k+2}}\left\|\left(1-T_{\epsilon}^{*}\right)^{-1}\right\|{ }_{o p}\left\|\epsilon\left(R_{\epsilon} T\right)^{*} v_{0}\right\|_{L^{2}} \\
& \leq c \epsilon^{-\frac{1}{2 k+2}}\left(1-\left\|T_{\epsilon}\right\|_{o p}\right)^{-1}\left\|\epsilon\left(R_{\epsilon} T\right)^{*} v_{0}\right\|_{L^{2}} \\
& \leq c \epsilon^{-\frac{1}{2 k+2}}\left\|\epsilon R_{\epsilon}^{*} v_{0}\right\|_{H^{-1}}
\end{aligned}
$$

Since $v_{0}(x)=c_{1} \delta(x)+c_{2} \delta(x-1)+w(x)$, with $c_{1} \neq 0$ and $w(x) \in C^{\infty}[0,1]$,

$$
\begin{aligned}
\left\|\epsilon R_{\epsilon}^{*} v_{0}(x)\right\|_{H^{-1}} & =\sup _{\|g\|_{H^{1}=1}}\left|\left(\epsilon R_{\epsilon}^{*} v_{0}(x), g\right)\right| \\
& \leq \sup _{\|g\|_{H^{1}=1}}\left|c_{2} \in R_{\epsilon} g(1)\right|+\left|\left(w, \in R_{\epsilon} g\right)\right| .
\end{aligned}
$$


Now we will show that $\left|\epsilon R_{\epsilon} g(1)\right| \leq c \epsilon$.

$$
\begin{aligned}
\left|\epsilon R_{\epsilon} g(1)\right| & =\left|\int_{0}^{1} \exp \left(-\int_{y}^{1} \frac{a(s)}{\epsilon} d s\right) g(y) d y\right| \\
& \leq\|g\|_{L^{\infty}} \int_{0}^{1} \exp \left(-c \frac{\left(1-y^{k+1}\right)}{\epsilon}\right) d y .
\end{aligned}
$$

Let

$$
\begin{aligned}
& I_{1}=\int_{1-\epsilon}^{1} \exp \left(-c \frac{1-y^{k+1}}{\epsilon}\right) d y \\
& I_{2}=\int_{0}^{1-\epsilon} \exp \left(-c \frac{1-y^{k+1}}{\epsilon}\right) d y
\end{aligned}
$$

Then it is easy to see that $I_{1} \approx c \epsilon$ and $I_{2} \approx<c \epsilon$.

We also have the estimate

$$
\begin{aligned}
\left|\left(w, \epsilon R_{\epsilon} g\right)\right| & <\|w\|_{L^{\infty}}\|g\|_{L^{\infty}} \int_{0}^{1} \int_{0}^{x} \exp \left(-\int_{y}^{x} \frac{a(s)}{\epsilon} d s\right) d y d x \\
& <\|w\|_{L^{\infty}}\|g\|_{L^{\infty}} \int_{0}^{1} \int_{0}^{x} \exp \left(-c \frac{x^{k+1}-y^{k+1}}{\epsilon}\right) d y d x
\end{aligned}
$$

Integrating over a small region where the integrand is near 1 and a larger region where the integrand is small, we get $\left|\left(w, \epsilon R_{\epsilon} g\right)\right| \leq c \epsilon^{3 / 2 k+2}$.

Therefore, $\left|\left(E_{\epsilon}(x, 0), v_{\epsilon}\right)\right|=c_{1}+O\left(\epsilon^{1 / k+1}\right)$, so $\left|\left(E_{\epsilon}(x, 0), v_{\epsilon}\right)\right|^{-1}=1 / c_{1}+O\left(\epsilon^{1 / k+1}\right)$.

Now we consider the numerator, $\left(R_{\epsilon} f, v_{\epsilon}(x)\right)$. By (1.7) $R_{\epsilon} f=\frac{f}{a}-\frac{f}{a}(0) E_{\epsilon}(x, 0)-\epsilon R_{\epsilon} \frac{d}{d x} \frac{f}{a}$. So

$$
\begin{aligned}
\left(\frac{f}{a}, v_{\epsilon}\right) & =\left(\frac{f}{a}, v_{0}\right)+\left(\frac{f}{a}, R_{0}^{*} h_{\epsilon}\right) \\
& =\left(\frac{f}{a}, v_{0}\right)+\left(R_{0} \frac{f}{a}, h_{\epsilon}\right)
\end{aligned}
$$

Now

$$
\left|\left(R_{0} \frac{f}{a}, h_{\epsilon}\right)\right| \leq\left\|R_{0} \frac{f}{a}\right\|_{L^{2}}\left\|h_{\epsilon}\right\|_{L^{2}} \leq c\left\|R_{0} \frac{f}{a}\right\|_{L^{2}} \epsilon^{1 / k+1}
$$

Also

$$
\begin{aligned}
\left|\left(\epsilon R_{\epsilon} \frac{d}{d x} \frac{f}{a}, v_{\epsilon}(x)\right)\right| & \leq\left|\left(\epsilon R_{\epsilon} \frac{d}{d x} \frac{f}{a}, v_{0}\right)\right|+\left|\left(\epsilon R_{\epsilon} \frac{d}{d x} \frac{f}{a}, R_{0}^{*} h_{\epsilon}\right)\right| \\
& \leq\left\|\frac{d}{d x} \frac{f}{a}\right\| L_{L^{2}}\left\|\epsilon R_{\epsilon}^{*} v_{0}\right\|_{L^{2}}+\left\|\epsilon R_{0} R_{\epsilon} \frac{d}{d x} \frac{f}{a}\right\| L_{L^{2}}\left\|h_{\epsilon}\right\|_{L^{2}} \\
& \leq c\left\|\frac{d}{d x} \frac{f}{a}\right\| L_{L^{2}} \epsilon^{1 / k+1}+c \epsilon^{1 / 2 k+2} \epsilon^{1 / k+1} \\
& \leq c \epsilon^{1 / k+1} .
\end{aligned}
$$

Hence, $c_{\epsilon}=\frac{f}{a}(0)-\left(\frac{f}{a}, v_{0}(x)\right) / c_{1}+O\left(\epsilon^{1 / k+1}\right)$. 
Theorem 1.1. Suppose that Assumption 1.1 holds, then there exists an $\epsilon_{0}>0$ such that for $0<\epsilon<\epsilon_{0}$, there exists a unique solution $u_{\epsilon}(x) \in L^{2}(0,1)$, which is given by

$$
u_{\epsilon}(x)=\left(I-T_{\epsilon}\right)^{-1} R_{0}\left(R_{\epsilon} f+c_{\epsilon} E_{\epsilon}(x, 0)\right)
$$

for each $f \in L^{2}(0,1)$.

Proof. Let $f \in L^{2}(0,1)$. Define $R_{0}$ by $(1.16), R_{\epsilon}$ by $(1.4), E_{\epsilon}(x, 0)$ by $(1.5)$, and $c_{\epsilon}$ by (1.24). By Lemmas $1.1-1.6$ for $\epsilon>0$ and sufficiently small, $u_{\epsilon}(x)$ is in $L^{2}(0,1)$. Now we will show that $u_{\epsilon}(x)$ is the solution of (1.2).

Apply $B_{\epsilon}=B-\epsilon R_{\epsilon} T$, defined by (1.11), to (1.25).

$$
B_{\epsilon} u_{\epsilon}(x)=B_{\epsilon}\left(I-T_{\epsilon}\right)^{-1} R_{0}\left(R_{\epsilon} f+c_{\epsilon} E_{\epsilon}(x, 0)\right) .
$$

By the choice (1.24) of $c_{\epsilon}$, we find

$$
\left(R_{\epsilon} f+c_{\epsilon} E_{\epsilon}(x, 0), v_{\epsilon}\right)=0 .
$$

Then

$$
B_{\epsilon} u_{\epsilon}(x)=R_{\epsilon} f+c_{\epsilon} E_{\epsilon}(x, 0) .
$$

Evaluating this expression at $x=0$, we get (1.13):

$$
B u_{\epsilon}(0)=\int_{0}^{1} Q(0, y) u_{\epsilon}(y) d y=c_{\epsilon} .
$$

Finally, apply $L_{\epsilon}$ to recover (1.2).

Theorem 1.2. Suppose Assumption 1.1 holds. If $f_{a} \in H^{1}[0,1]$ and orthogonal to the cokernel of $B$, then $u_{\epsilon}(x) \rightarrow u_{0}(x)$ in $L^{2}[0,1]$ as $\epsilon \rightarrow 0$.

Proof. By (1.25) and (1.7),

$$
\begin{aligned}
u_{\epsilon}(x) & =\left(1-T_{\epsilon}\right)^{-1} R_{0}\left(R_{\epsilon} f+c_{\epsilon} E_{\epsilon}(x, 0)\right) \\
& =\left(1-T_{\epsilon}\right)^{-1} R_{0}\left(\frac{f}{a}-\frac{f}{a}(0) E_{\epsilon}(x, 0)-\epsilon R_{\epsilon} \frac{d}{d x} \frac{f}{a}+c_{\epsilon} E_{\epsilon}(x, 0)\right)
\end{aligned}
$$

By Lesmma 1.6,

$u_{\epsilon}(x)=\left(1-T_{\epsilon}\right)^{-1} R_{0}\left(\frac{f}{a}-\frac{f}{a}(0) E_{\epsilon}(x, 0)-\epsilon R_{\epsilon} \frac{d}{d x} \frac{f}{a}+\left(\frac{f}{a}(0)-\left(\frac{f}{a}, v_{0}\right) / c_{1}+O\left(\epsilon^{1 / k+1}\right)\right) E_{\epsilon}(x, 0)\right)$.

By the orthogonality condition $\left(\frac{f}{a}, v_{0}\right)=0$. By Lemma $1.2,\left\|R_{0} E_{\epsilon}(x, 0)\right\| \leq c \epsilon^{-1 / 2 k+2}$ and Lemma 1.5 implies that for $\epsilon$ sufficiently small $\left(1-T_{\epsilon}\right)^{-1}=\sum_{j=0}^{\infty} T_{\epsilon}^{j}$ converges in operator norm and $\left\|T_{\epsilon}^{l} w\right\|_{L^{2}} \rightarrow 0$ in $L^{2}$ for $l=1,2,3$. So $\left\|u_{\epsilon}(x)\right\|_{L^{2}} \leq\left\|u_{0}(x)\right\|_{L^{2}}+c \epsilon^{1 / 2 k+2}$ and $u_{\epsilon}(x) \rightarrow u_{0}(x)=R_{0} \frac{f}{a}$ in $L^{2}$ as $\epsilon \rightarrow 0$. 
Corollary 1.2. Suppose Assumption 1.1 holds. If $\frac{f}{a} \in H^{1}[0,1]$ but not orthogonal to the cokernel of $B$, then for $\epsilon$ sufficiently small, $\left\|u_{\epsilon}(x)\right\|_{L^{2}} \geq c \epsilon^{-\frac{1}{2 k^{3}+2}}$.

Proof. Since $\frac{f}{a}$ is not orthogonal to the cokernel of $B,\left(\frac{f}{a}, v_{0}\right) \neq 0$. From (1.26), we see $\left\|u_{\epsilon}(x)\right\|_{L^{2}} \geq-C+\left\|\left(\frac{f}{a}, v_{0}\right) / c_{1} R_{0} E_{\epsilon}(x, 0)\right\|_{L^{2}}+c \epsilon^{1 / 2 k+2}$. Lemma 1.2 is sharp in the sense that $\left\|\frac{d}{d x} E_{\epsilon}(x, 0)\right\|_{L^{2}} \approx c \epsilon^{-1 / 2 k+2}$. So for $\epsilon$ sufficiently small, we have $\left\|u_{\epsilon}(x)\right\|_{L}^{2} \geq$ $c \epsilon^{-1 / 2 k+2}$.

There is a boundary layer at $x=0$. It can be shown that if $\frac{f}{a} \in H^{1}[0,1]$ and orthogonal to the cokernel of $B$, then $u_{\epsilon}(x) \rightarrow u_{0}(x)$ uniformly as $\epsilon \rightarrow 0$ on compact subsets of $[0,1]$ not containing the origin. Also since it can be shown that $R_{0} E_{\epsilon}(x, 0) \rightarrow 0$ uniformly as $\epsilon \rightarrow 0$ on closed subsets of $[0,1]$ not containing the origin, it can be shown the if $\frac{f}{a} \in H^{2}$ but is not orthogonal to the cokernel of $B$ then $u_{\epsilon}(x) \rightarrow u_{0}(x)$ as $\epsilon \rightarrow 0$ uniformly on closed subsets of $[0,1]$ not containing the origin.

\section{Remark:}

The solution, $u_{\epsilon}(x)$, has complicated behavior as $\epsilon \rightarrow 0$ if $f / a$ is not in $H^{1}$. The reason is that $R_{0}$ contains terms which are integral operators while $f / a$ is singular at the origin, which means that $R_{0} R_{\epsilon} f$ may be undefined as $\epsilon \rightarrow 0$. However, Theorem 1.1 is true for any $f \in L^{2}$ for $\epsilon>0$. These results are in marked constrast with those of the non-degenerate case discussed in [S] (c.f. also [E3]).

2. Degeneracy in an interior point with $k$ odd: reduction to a regular perturbation problem.

We sketch the case of

$$
\epsilon u_{\epsilon}(x)+a(x)\left(\int_{-1}^{x} u_{\epsilon}(y) d y+\int_{-1}^{1} Q(x, y) u_{\epsilon}(y) d y\right)=f(x)
$$

where $a(x)=x^{k} b(x), k$ is an odd positive integer, $b(x) \in C^{\infty}[-1,1]$ and $b(x) \geq c>0$. The kernel $Q(x, y)$ has the same form as $(1.1)$ but is defined on $[-1,1] \times[-1,1]$.

The associated differential operator $L_{\epsilon},(1.3)$, is now defined on $(-1,1)$ and $R_{\epsilon}$ is given by

$$
\begin{aligned}
R_{\epsilon} f & =\int_{0}^{x} \frac{E_{\epsilon}(x, y)}{\epsilon} f(y) d y, \\
E_{\epsilon}(x, y) & =\exp \left(-\int_{y}^{x} \frac{a(s)}{\epsilon}\right) d s .
\end{aligned}
$$

The general relations $(1.6 \mathrm{a}, 6 \mathrm{~b})$ hold for $w \in H^{1}[-1,1]$ and $g \in L^{2}(-1,1)$.

We apply $R_{\epsilon}$ to (2.1) to obtain

$$
\begin{aligned}
R_{\epsilon} f= & \int_{-1}^{x} u_{\epsilon}(y) d y+\int_{-1}^{1} Q(x, y) u_{\epsilon}(y) d y \\
& -\epsilon R_{\epsilon} \int_{-1}^{1} \frac{\partial Q}{\partial x}(x, y) u_{\epsilon}(y) d y-E_{\epsilon}(x, 0)\left(\int_{-1}^{0} u_{\epsilon}(y) d y+\int_{-1}^{1} Q(0, y) u_{\epsilon}(y) d y\right) \\
& :=B_{\epsilon} u_{\epsilon}(x)-c_{\epsilon} E_{\epsilon}(x, 0) .
\end{aligned}
$$


Set

$$
c_{\epsilon}=\int_{-1}^{0} u_{\epsilon}(y) d y+\int_{-1}^{1} Q(0, y) u_{\epsilon}(y) d y .
$$

We will treat $c_{\epsilon}$ as an unspecified constant to be determined shortly.

The associated reduced equation to $(2.1)$ is

$$
\begin{aligned}
B u_{0} & =\int_{-1}^{x} u_{0}(x)(y) d y+\int_{-1}^{1} Q(x, y) u_{0}(x)(y) d y \\
& =g
\end{aligned}
$$

where $g \in H^{1}[-1,1]$.

Let $($,$) denote the natural pairing between H^{1}(-1,1)$ and its dual $H^{-1}(-1,1)$.

Assumption 2.1: $B$ has a one dimensional cokernel, spanned by $v_{0}(x)$, which means $\left(B u, v_{0}(x)\right)=0$, and $v_{0}(x)$ has the form

$$
v_{0}(x)=c_{1} \delta(x+1)+c_{2} \delta(x-1)+w(x)
$$

where we further assume that $w(0) \neq 0$.

The function $w$ will be in $C^{\infty}[-1,1]$, by the argument of Lemma 1.1.

The assumption implies that there exists $R_{0}$ such that $R_{0} B=I$. We will call the reduced solution $u_{0}(x)=R_{0} g$ if $g \in H^{1}$ and is orthogonal to the cokernel of $B$.

Let

$$
T_{\epsilon} w=\epsilon R_{0} R_{\epsilon} \int_{-1}^{1} \frac{\partial Q}{\partial x}(x, y) w(y) d y \quad w \in L^{2}(-1,1)
$$

Apply $R_{0}$ to (2.2) to obtain

$$
\left(I-T_{\epsilon}\right) u_{\epsilon}(x)=R_{0}\left(R_{\epsilon} f+c_{\epsilon} E_{\epsilon}(x, 0)\right)
$$

Lemma 2.1. $T_{\epsilon}$ is a bounded operator from $L^{2}[-, 1,1]$ to $H^{1}[-1,1]$ which satisfies the estimate $\left\|T_{\epsilon} w\right\|_{L^{2}} \leq c \epsilon^{1 / 2 k+1}\|w\|_{H^{1}}$.

Proof. The proof follows from Lemma 1.5 and the observation that when $c_{1} x^{k} \leq a(x) \leq$ $c_{2} x^{k}$, so $d_{1}\left(x^{k+1}-y^{k+1}\right) \leq E_{\epsilon}(x, y) \leq d_{2}\left(x^{k+1}-y^{k+1}\right)$ and a similar estimate holds for $E_{\epsilon}(-x,-y)$ since $k$ is an odd integer.

Therefore for $\epsilon$ sufficiently small

$$
u_{\epsilon}(x)=\left(I-T_{\epsilon}\right)^{-1} R_{0}\left(R_{\epsilon} f+c_{\epsilon} E_{\epsilon}(x, 0)\right) .
$$

For the same reasons as in $\S 1$, we pick

$$
c_{\epsilon}=-\frac{\left(R_{\epsilon} f, v_{\epsilon}\right)}{\left(E_{\epsilon}(x, 0), v_{\epsilon}\right)}
$$


where $\left(B_{\epsilon} u_{1} v_{\epsilon}\right)=0$ for all $u \in L^{2}(-1,1)$ and

$$
v_{\epsilon}=v_{0}(x)+R_{0}^{*}\left(I-T_{\epsilon}^{*}\right)^{-1} \epsilon\left(R_{\epsilon} T\right)^{*} v_{0}(x)
$$

We claim that when Assumption 2.1 holds, $c_{\epsilon}=-\frac{\left(\frac{f}{a}, v_{0}\right)}{d_{1}} \epsilon^{-1 / k+1}+\frac{f}{a}(0)+O\left(\epsilon^{1 / k+1}\right)$ by an argument similar to the one given in Lemma 1.6. However, the denominator $\left(E_{\epsilon}(x, 0), v_{\epsilon}\right)$ is composed of two exponentially small terms $c_{1} E_{\epsilon}(1,0)$ and $c_{2} E_{\epsilon}(-1,0)$ and by Assumption 2.1 there exist $d_{1}$ and $d_{2}$ such that

$$
d_{1} \epsilon^{1 / k+1} \leq\left|\left(E_{\epsilon}(x, 0), w\right)\right| \leq d_{2} \epsilon^{1 / k+1} .
$$

So for $\epsilon$ sufficiently small, $\left(E_{\epsilon}(x, 0), w\right)$ is the dominant term.

By arguments analogous to those given in Theorems 1.1 and 1.2, we can state the following theorems:

Theorem 2.1. If Assumption 2.1 holds, then there exists an $\epsilon_{0}>0$ such that for $0<\epsilon<$ $\epsilon_{0}$, there exists a unique solution in $L^{2}(-1,1)$ to (2.1) given by $(2.3)$ for each $f \in L^{2}(-1,1)$.

Theorem 2.2. If Assumption 2.1 holds and if $\frac{f}{a} \in H^{1}[-1,1]$ and orthogonal to the cokernel of $B$, then $u_{\epsilon} \rightarrow u_{0}=R_{0} \frac{f}{a}$ in $L^{2}$ as $\epsilon \rightarrow 0$.

Corollary 2.2. If Assumption 2.1 holds and if $\frac{f}{a} \in H^{1}[-1,1]$ but is not orthogonal to the cokernel of $B$, then $\left\|u_{\epsilon}(x)\right\|_{L^{2}} \geq-C+c \epsilon^{-3 / 2 k+2}$.

3. Degeneracy at an interior point with $k$ even: reduction to a regular perturbation problem.

We sketch the case of the singularly perturbed integral equation

$$
\epsilon u_{\epsilon}(x)+a(x)\left(\int_{-1}^{x} u_{\epsilon}(y) d y+\int_{-1}^{1} Q(x, y) u_{\epsilon}(y) d y\right)=f(x)
$$

where $a(x)=x^{k} b(x)$, where $k$ is an even positive integer and $b(x) \geq c>0, b(x) \in$ $C^{\infty}[-1,1]$. The kernel $Q(x, y)$ is the same as in $\S 2$. We define the operator $L_{\epsilon}$ and $R_{\epsilon}$ :

$$
\begin{array}{ll}
L_{\epsilon} w(x)=\epsilon \frac{d}{d x} w(x)+a(x) w(x) & w \in H^{1}[-1,1], \\
R_{\epsilon} f(x)=\int_{-1}^{x} \frac{E_{\epsilon}(x, y)}{\epsilon} f(y) d y & f \in L^{2}(-1,1)
\end{array}
$$

which satisfy the general relations:

$$
\begin{aligned}
& R_{\epsilon} L_{\epsilon} w(x)=w(x)-w(-1) E_{\epsilon}(x,-1) \\
& L_{\epsilon} R_{\epsilon} f(x)=f(x)
\end{aligned}
$$


The reduced equation is given by

$$
B u_{0}=\int_{-1}^{x} u_{0}(y) d y+\int_{-1}^{1} Q(x, y) u_{0}(y) d y=g
$$

where $g \in H^{1}[-1,1]$.

By an argument similar to Lemma 1.1, the elements of the cokernel of $B$, ie the $v_{0} \in H^{-1}$ with $\left(B u, v_{0}(x)\right)=0$ for all $u$, have the form

$$
v_{0}(x)=c_{1} \delta(x+1)+c_{2} \delta(x-1)+w(x)
$$

with $w \in C^{\infty}[0,1]$.

Assumption 3.1: B has a one dimensional cokernel, and the constant $c_{1}$ is nonzero.

Let $R_{0}$ denote an operator such that $R_{0} B=I$ and $B_{\epsilon}$ be of the form (2.2). As before we will call the reduced solution $u_{0}(x)=R_{0} g$ when $g \in H^{1}$ and orthogonal to the cokernel of $B$.

Applying $R_{0} R_{\epsilon}$ to (3.1). we obtain

$$
u_{\epsilon}(x)+\epsilon R_{0} R_{\epsilon} \int_{-1}^{1} \frac{\partial Q}{\partial x}(x, y) u_{\epsilon}(y) d y=R_{0}\left(R_{\epsilon} f+c_{\epsilon} E_{\epsilon}(x,-1)\right)
$$

where

$$
c_{\epsilon}=\int_{-1}^{1} Q(-1, y) u_{\epsilon}(y) d y
$$

is an unspecified constant to be determined later on. Set

$$
T_{\epsilon} w=\epsilon R_{0} R_{\epsilon} \int_{-1}^{1} \frac{\partial Q}{\partial x}(x, y) w(y) d y \quad w \in L^{2}(-1,1) .
$$

It can be shown that $T_{\epsilon}$ is a bounded operator from $L^{2}[-1,1]$ into $H^{1}[-1,1]$ which satisfies the estimate

$$
\left\|T_{\epsilon} w\right\|_{L^{2}} \leq c \epsilon^{\frac{1}{2 k+2}}\|w\|_{H^{1}}
$$

by a similar argument to the one in the proof of Lemma 1.5.

Then for $\epsilon$ sufficiently small

$$
u_{\epsilon}(x)=\left(I-T_{\epsilon}\right)^{-1} R_{0}\left(R_{\epsilon} f+c_{\epsilon} E_{\epsilon}(x,-1)\right) .
$$

The crucial step is to define

$$
c_{\epsilon}=-\frac{\left(R_{\epsilon} f, v_{\epsilon}\right)}{\left(E_{\epsilon}(x,-1), v_{\epsilon}\right)}
$$

where $\left(B_{\epsilon} u, v_{\epsilon}\right)=0$ for all $u \in L^{2}(-1,1)$ and

$$
\begin{aligned}
& v_{\epsilon}(x)=v_{0}(x)+R_{0}^{*} h_{\epsilon}, \\
& h_{\epsilon}=\left(I-T_{\epsilon}^{*}\right)^{-1} \epsilon\left(R_{0}^{*} T\right)^{*} v_{0}(x) .
\end{aligned}
$$

As in Lemma 1.6, we have $c_{\epsilon}=-\frac{\left(\frac{f}{a}, v_{0}(x)\right)}{c_{2}}+\frac{f}{a}(-1)+O\left(\epsilon^{1 / k+1}\right)$ provided that Assumption 3.1 holds.

By arguments analogous to those given in Theorem 1.1 and Theorem 1.2 , we can state the following theorems: 
Theorem 3.1. If Assumption 3.1 holds, then there exists an $\epsilon_{0}>0$, such that for $0<\epsilon<$ $\epsilon_{0}$, there exists a unique $L^{2}(-1,1)$ solution to (3.1) given by (3.3) for each $f \in L^{2}(-1,1)$.

Theorem 3.2. If Assumption 3.1 holds and if $\frac{f}{a} \in H^{1}[-1,1]$ and orthogonal to the cokernel of $B$, then $u_{\epsilon} \rightarrow u_{0}=R_{0} \frac{f}{a}$ in $L^{2}$ as $\epsilon \rightarrow 0$.

Corollary 3.2. If Assumption 3.1 holds and if $\frac{f}{a} \in H^{1}[-1,1]$ but is not orthogonal to the cokernel of $B$, then $\left\|u_{\epsilon}(x)\right\|_{L^{2}} \geq-C+c \epsilon^{-1 / 2 k+2}$.

\section{A sign change in the degeneracy at an interior point with $k$ odd.}

In this section we are examine the dual situation of the problem discussed in $\S 2$. Suppose we are given $f \in L^{2}(-1,1)$. We consider the singularly perturbed integral equation

$$
\epsilon u_{\epsilon}(x)-\int_{-1}^{x} a(y) u_{\epsilon}(y) d y-\int_{-1}^{1} a(y) Q(x, y) u_{\epsilon}(y) d y=f(x)
$$

where $a(x)=x^{k} b(x), b(x) \in C^{\infty}[-1,1], b(x)>0$ and $k$ is an odd positive integer. The kernel $Q(x, y)$ is the same as in $\S 2$.

We find sufficient conditions for the existence and uniqueness of an $L^{2}(-1,1)$ solution to (4.1). First we extend (4.1) to $\mathbf{R}$ in the following manner. Denote still by $u_{\epsilon}(x)$, $u_{\epsilon}(x) \in L^{2}(\mathbf{R})$, the zero extension of $u_{\epsilon}(x) \in L^{2}(-1,1)$, i.e. the new $u_{\epsilon}$ is the same as the original $u_{\epsilon}$ for $x \in(-1,1)$ and zero otherwise. Let $f_{+}(x)$ be the zero extension of $f$ as an element in $L^{2}(\mathbf{R})$. Let $p$ denote the restriction operator to $(-1,1)$, then $p f_{+}(x)=f$. Take smooth bounded extensions of $Q(x, y)$ and $a(x)$.

Then (4.1) becomes

$$
\epsilon u_{\epsilon}(x)-\int_{-\infty}^{x} a(y) u_{\epsilon}(y) d y-\int_{-\infty}^{\infty} a(y) Q(x, y) u_{\epsilon}(y) d y=f_{+}(x)
$$

Let $\stackrel{\circ}{H}_{s}(-1,1)$ denote the space of distributions which are in $H_{s}(R)$ with support contained in $[-1,1]$. We define the operators $L_{\epsilon}, R_{\epsilon}$ by

$$
\begin{gathered}
L_{\epsilon} w=\epsilon \frac{d}{d x} w-a(x) w \quad w \in \stackrel{\circ}{H}_{0}(-1,1) \\
R_{\epsilon} g=-\Theta(x) \int_{x}^{1} \frac{E_{\epsilon}(x, y)}{\epsilon} g(y) d y+\Theta(-x) \int_{-1}^{x} \frac{E_{\epsilon}(x, y)}{\epsilon} g(y) d y \\
g \in \stackrel{\circ}{H}_{-1}(-1,1)
\end{gathered}
$$

where

$$
E_{\epsilon}(x, y)=\exp \left(\int_{y}^{x} \frac{a(s)}{\epsilon} d s\right)
$$

It is easy to verify the general relations:

$$
\begin{aligned}
& R_{\epsilon} L_{\epsilon} w(x)=w(x), \\
& L_{\epsilon} R_{\epsilon} g(x)=g(x) \quad \text { if }\left(g, E_{\epsilon}(0, x)\right)=0
\end{aligned}
$$


Set

$$
T u_{\epsilon}(x)=p \int_{-\infty}^{\infty} Q(x, y) \frac{d}{d y} u_{\epsilon}(y) d y
$$

Now we can better see the structure of (4.1) by writing it as

$$
p B L_{\epsilon} u_{\epsilon}+T u_{\epsilon}=f
$$

The associated reduced equation of (4.1) is

$$
\begin{aligned}
p B v & =\int_{-\infty}^{x} v(y) d y+\int_{-\infty}^{\infty} Q(x, y) v(y) d y \\
& =f
\end{aligned}
$$

where $v=a u_{0}$.

Since $p B$ has index 1 as an operator from $\stackrel{\circ}{H}_{-1}(-1,1)$ to $L^{2}(-1,1)$, the following assumption on $p B$ is natural as it is nothing more than the dual situation compared with $\S 2$.

Assumption 4.1: The operator $p B: \stackrel{\circ}{H}_{-1}(-1,1) \rightarrow L^{2}(-1,1)$ in $(4.7)$ has a one dimensional kernel.

Let $k(x)$ span the kernel of $p B$, i.e. $p B k(x)=0 . k(x)$ has the form

$$
k(x)=c_{1} \delta(x+1)+c_{2} \delta(x-1)+w(x)
$$

where $w(x) \in C^{\infty}[-1,1]$, by an argument similar to Lemma 1.1 .

Assumption 4.2: We assume that the smooth term in the kernel of $p B(4.8)$ does not vanish at the origin, i.e. $w(0) \neq 0$.

Let $R_{0}$ be the operator with the following properties:

$$
R_{0}: L^{2}(-1,1) \rightarrow \stackrel{\circ}{H}_{-1}(-1,1): \quad \quad p B R_{0} g=g
$$

provided that $R_{0} g$ is not in the kernel of $p B$ and

$$
R_{0} p B v_{0}(x)=v_{0}(x)+c k(x)
$$

where $c$ is an arbitrary constant.

Then if $R_{0} f$ is not in the kernel of $p B$, the solution to the reduced equation (4.7) is $u_{0}=\frac{R_{0} f}{a}$.

After applying $R_{0}$ to (4.6), we have

$$
L_{\epsilon} u_{\epsilon}(x)-\epsilon R_{0} T u_{\epsilon}(x)=R_{0} f+c_{\epsilon} k(x) .
$$


We will determine $c_{\epsilon}$ by requiring that the compatibility condition (4.4) should be satisfied:

$$
\left(R_{0} f+c_{\epsilon} k(x)+\epsilon R_{0} T u_{\epsilon}(x), E_{\epsilon}(0, x)\right)=0 .
$$

Thus, we take

$$
\mathcal{c}_{\epsilon}=-\left(k(x), E_{\epsilon}(0, x)\right)^{-1}\left(\epsilon R_{0} T u_{\epsilon}(x)+R_{0} f, E_{\epsilon}(0, x)\right)
$$

and decompose it as a sum of two terms,

$$
c_{\epsilon}=c_{1 \epsilon}+c_{2 \epsilon}
$$

where

$$
\begin{aligned}
& c_{1 \epsilon}=-\left(k(x), E_{\epsilon}(0, x)\right)^{-1}\left(\epsilon R_{0} T u_{\epsilon}(x), E_{\epsilon}(0, x)\right), \\
& c_{2 \epsilon}=-\left(k(x), E_{\epsilon}(0, x)\right)^{-1}\left(R_{0} f, E_{\epsilon}(0, x)\right) .
\end{aligned}
$$

We see that $c_{1 \epsilon}$ depends on the unknown function $u_{\epsilon}(x)$. It will be determined in terms of $f$ shortly.

Apply $R_{\epsilon}$ to $(4.10)$ to obtain

$$
u_{\epsilon}(x)-\epsilon R_{\epsilon} R_{0} T u_{\epsilon}(x)=R_{\epsilon}\left(R_{0} f+c_{\epsilon} k(x)\right)
$$

In order to prove that (4.13) can be viewed is a regular perturbation problem, we will show that

$$
T_{\epsilon} u_{\epsilon}(x):=R_{\epsilon}\left(\epsilon R_{0} T u_{\epsilon}(x)+c_{1 \epsilon} k(x)\right)
$$

has small norm in $\stackrel{\circ}{H}_{0}(-1,1)$.

Lemma 4.1. $T_{\epsilon}$ defined by (4.14) is a bounded operator from $\stackrel{\circ}{H}_{0}(-1,1)$ into $\stackrel{\circ}{H}_{0}(-1,1)$ which satisfies the estimate

$$
\left\|T_{\epsilon} w\right\|_{{\stackrel{\circ}{H_{0}}}_{0}(-1,1)} \leq c \epsilon^{\frac{1}{2 k+2}}\|w\|_{\stackrel{\circ}{H}_{0}(-1,1)}
$$

Proof. The first term in (4.14) satisfies the estimate

$$
\left\|\epsilon R_{\epsilon} R_{0} T w\right\|_{\stackrel{\circ}{H}(-1,1)_{0}} \leq c \epsilon^{1 / k+1}\|w\|_{\stackrel{\circ}{H}_{0}(-1,1)}
$$

which may be established by the similar argument as in the proof of Lemma 1.4. Let us have a look of the second term. Since

$$
k(x)=c_{1} \delta(x+1)+c_{2} \delta(x-1)+w(x),
$$


by Assumption 4.2, there exist $\beta_{1}, \beta_{2}$ such that

$$
\beta_{1} \epsilon^{1 / k+1} \leq\left|\left(k(x), E_{\epsilon}(0, x)\right)\right| \leq \beta_{2} \epsilon^{1 / k+1}
$$

It is easy to see that

$$
\begin{aligned}
\left|\left(R_{0} T w, E_{\epsilon}(0, x)\right)\right| & =\left|\left(T w, R_{0}^{*} E_{\epsilon}(0, x)\right)\right| \\
& \leq c \epsilon^{\frac{1}{2 k+2}}\|w\|_{\stackrel{\circ}{0}_{0}(-1,1)}
\end{aligned}
$$

Thus, by estimates similar to those given in the proof of Lemma 1.3, we have

$$
\left\|R_{\epsilon} k(x)\right\|_{\stackrel{\circ}{H}_{0}(-1,1)} \leq c \epsilon^{-\frac{k}{k+1}}\|w\|_{\stackrel{\circ}{H}_{0}(-1,1)} .
$$

Combining (4.16), (4.17), (4.18), and (4.19), we get (4.15).

Thus for $\epsilon$ sufficiently small, $\left(I-T_{\epsilon}\right)^{-1}$ exists and

$$
u_{\epsilon}(x)=\left(I-T_{\epsilon}\right)^{-1} R_{\epsilon}\left(R_{0} f-c_{2 \epsilon} k(x)\right) .
$$

Now we substitute (4.21) into (4.12) to obtain

$$
c_{1 \epsilon}=-\left(k(x), E_{\epsilon}(0, x)\right)^{-1}\left(\epsilon R_{0} T\left(I-T_{\epsilon}\right)^{-1} R_{\epsilon}\left(R_{0} f-c_{2 \epsilon} k(x)\right), E_{\epsilon}(0, x)\right)
$$

Lemma 4.2. When Assumption 4.2 holds, $c_{\epsilon}$ is well defined and $O\left(\epsilon^{-\frac{k}{k+1}}\right)$.

Proof. We write $c_{\epsilon}=c_{1 \epsilon}+c_{2 \epsilon}$, where $c_{1 \epsilon}, c_{2 \epsilon}$ are given by (4.21) and (4.12), respectively. Let us examine $c_{2 \epsilon}$ first. By Assumption 4.2, the numerator satisfies the following inequality:

$$
\begin{aligned}
\left|\left(R_{0} f, E_{\epsilon}(0, x)\right)\right| & \leq c\left\|R_{0} f\right\|_{H_{-1}(-1,1)}\left\|E_{\epsilon}(0, x)\right\|_{H_{1}(-1,1)} \\
& \leq c \epsilon^{-\frac{1}{2 k+2}}
\end{aligned}
$$

Therefore, $c_{2 \epsilon}=O\left(\epsilon^{-\frac{1}{k+1}}\right)$.

Now we consider $c_{1 \epsilon}$. The denominator of $c_{1 \epsilon}$ also satisfies (4.17). The numerator is composed of two terms:

$$
\begin{aligned}
& A_{1}=\epsilon c_{2 \epsilon}\left(R_{0} T\left(I-T_{\epsilon}\right)^{-1} R_{\epsilon} k, E_{\epsilon}(0, x)\right), \\
& A_{2}=\epsilon\left(R_{0} T\left(I-T_{\epsilon}\right)^{-1} R_{\epsilon} R_{0} f_{1} E_{\epsilon}(0, x)\right)
\end{aligned}
$$

Using our assumption that $k(x)$ has the form (4.8), we have

$$
\begin{aligned}
\left|A_{1}\right| & \leq c \epsilon c_{2 \epsilon}\left|\left(R_{\epsilon} k(x), E_{\epsilon}(0, x)\right)\right| \\
& \leq c \epsilon c_{2 \epsilon}\left|\left(R_{\epsilon} w(x), E_{\epsilon}(0, x)\right)\right|
\end{aligned}
$$


because $R_{\epsilon} \delta(x \pm 1)=0$. Thus,

$$
\begin{aligned}
\left|A_{1}\right| & \leq c \epsilon c_{2 \epsilon}\left\|R_{\epsilon} w\right\|_{\stackrel{\circ}{H}(-1,1)_{0}}\left\|E_{\epsilon}(0, x)\right\|_{\mathscr{H}_{0}(-1,1)} \\
& \leq c \epsilon^{\frac{1}{2 k+2}}
\end{aligned}
$$

since

$$
\begin{aligned}
& \left\|R_{\epsilon} w\right\|_{\stackrel{\circ}{\circ}_{0}(-1,1)} \leq c \epsilon^{-\frac{k}{k+1}} \\
& \left\|E_{\epsilon}(0, x)\right\| \leq c \epsilon^{\frac{1}{2 k+2}}
\end{aligned}
$$

Finally, we also make an estimate on $A_{2}$.

$$
\begin{aligned}
\left|A_{2}\right| & \leq c \epsilon\left|\left(R_{\epsilon} R_{0} f_{1} E_{\epsilon}(0, x)\right)\right| \\
& \leq c \epsilon\left\|R_{\epsilon} R_{0} f\right\|_{\mathscr{O}_{0}(-1,1)}\left\|E_{\epsilon}(0, x)\right\|_{L^{2}(-1,1)} \\
& \leq c \epsilon^{-\frac{k}{k+1}} \epsilon^{\frac{1}{2 k+2}} .
\end{aligned}
$$

Therefore, $c_{\mathrm{I} \epsilon}=O\left(\epsilon^{-\frac{k}{k+1}}\right)$ which implies that $c_{\epsilon}=O\left(\epsilon^{-\frac{k}{k+1}}\right)$. Thus, the lemuma is proved.

Remark: Assumption 4.2 was necessary to ensure that the main term in the denominator of $c_{\epsilon}$ satisfies (4.17). If $w(x)$ vanishes to higher order, say $w^{j}(0)=0, w^{j+1}(0) \neq 0$, then the denominator of $c_{\epsilon}$ is $O\left(\epsilon^{\frac{2 i+1}{2 t+2}}\right)$. In the case when $Q(x, y)=0$, then $w(x)=0$, and the denominator is exponentially small which means that $c_{\epsilon}$ grows exponentially as $\epsilon \rightarrow 0$. In fact, it is not well defined if $c_{1} E_{\epsilon}(0,1)=c_{2} E_{\epsilon}(0,-1)$.

Theorem 4.1. Suppose Assumptions 4.1 and 4.2 hold, then there exists an $\epsilon_{0}$ such that for $0<\epsilon<\epsilon_{0}$, (4.1) has a unique solution $u_{\epsilon}(x) \in \stackrel{\circ}{H}_{0}(-1,1)$, which is given by (4.20) for each $f \in L^{2}(-1,1)$.

Proof. Let $f \in L^{2}(-1,1)$. Suppose Assumption 4.1 and 4.2 hold and $c_{\epsilon}=c_{1 \epsilon}+c_{2 \epsilon}$ where $c_{1 \epsilon}$ and $c_{2 \epsilon}$ are given by (4.11) and (4.21), respectively. Lemma 4.1 shows that for $\epsilon$ sufficiently small, $\left(I-T_{\epsilon}\right)^{-1}$ exists. Let $u_{\epsilon}(x)$

$$
u_{\epsilon}(x)=\left(I-T_{\epsilon}\right)^{-1} R_{\epsilon}\left(R_{0} f+c_{2 \epsilon} k(x)\right)
$$

where $T_{\epsilon}, R_{\epsilon}, k(x)$, and $R_{0}$ are given by (4.19), (4.3), (4.8), and (4.9), respectively. Then

$$
\left(I-T_{\epsilon}\right) u_{\epsilon}(x)=R_{\epsilon}\left(R_{0} f+c_{2 \epsilon} k(x)\right)
$$

and

$$
\begin{aligned}
u_{\epsilon}(x) & =T_{\epsilon} u_{\epsilon}(x)+R_{\epsilon}\left(c_{2 \epsilon} k(x)+R_{0} f\right) \\
& =\epsilon R_{\epsilon} R_{0} T u_{\epsilon}(x)-\left(k(x), E_{\epsilon}(0, x)\right)^{-1}\left(\epsilon R_{0} T u_{\epsilon}(x), E_{\epsilon}(0, x)\right) R_{\epsilon} k(x) \\
& +R_{\epsilon}\left(c_{2 \epsilon} k(x)+R_{0} f\right) .
\end{aligned}
$$


Apply $L_{\epsilon}$, given by (4.3), to the above expression, to obtain

$$
\begin{aligned}
L_{\epsilon} u_{\epsilon}(x) & =\epsilon R_{0} T u_{\epsilon}(x)+c_{2 \epsilon} k(x)+R_{0} f \\
& -\left(k(x), E_{\epsilon}(0, x)\right)^{-1}\left(\epsilon R_{0} T u_{\epsilon}(x), E_{\epsilon}(0, x)\right) k(x) .
\end{aligned}
$$

The compatibility condition (4.4) is satisfied by our choice of $c_{\epsilon}$, i.e.

$$
\begin{aligned}
& \left(\epsilon R_{0} T u_{\epsilon}(x)-\left(k(x), E_{\epsilon}(0, x)\right)^{-1}\left(\epsilon R_{0} T u_{\epsilon}(x), E_{\epsilon}(0, x)\right) k(x)\right. \\
& \left.\quad+c_{2 \epsilon} k(x)+R_{0} f, E_{\epsilon}(\mathbf{0}, x)\right)=\mathbf{0}
\end{aligned}
$$

Composing $p B,(4.7)$ with $L_{\epsilon}(4.22)$ to obtain

$$
p B L_{\epsilon} u_{\epsilon}(x)=\epsilon T u_{\epsilon}(x)-f,
$$

since by Assumption 4.1, $p B k(x)=0$. Thus, we have recovered (4.1),

$$
p B L_{\epsilon} u_{\epsilon}(x)-\epsilon T u_{\epsilon}(x)=f .
$$

Hence, the theorem is proved.

\section{REFERENCES}

[D] A. Demidov, Elliptic pseudodifferential boundary problems with a small parameter in the coefficient of the leading operator, Math. USSr Sb. 20 (1973), 439-463.

[E1] G. Eskin, Asymptotics of solutions of elliptic pseudodifferential equations with a small parameter, Dokl. Akad. Nauk USSR 211 (1973), 547-550, English translation Soviet Mathematics Doklady 14 (1973), 1080-1083.

[E2] _ Boundary Value Problems for Elliptic Pseudodifferential Equations, American Mathematical Society, Providence, 1981.

[E3] _ Degenerate elliptic pseudodifferential equations of principal type, Math. USSR Sbornik 11 (1970), 539-581.

[EV] G. Eskin and M. Višik, Convolution equations of variable order, Trudy Moskov, Math. Obšč 16 (1967), 25-50, English translation Trans. Moscow Math. Soc. (1967), 27-52.

[F] L. S. Frank, Coercive singular perturbations I: a priori estimates, Ann. Mat. Pura Appl. 119 (1979), 41-113.

[FW1] L. S. Frank and W. D. Wendt, Coercive singular perturbations II: reduction to regular perturbations and applications, Comm. Part. Diff. Eqn 7 (1982), 469-535.

[FW2] _ Coercive singular perturbations II: reduction and convergence, J. Math Anal. Appl. 88 (1982), 463-535.

[FW3] _ Coercive singular perturbations III: Wiener-Hopf operators, J. d'Analyse Math. 43 (1984), 88-135.

[G] G. Grubb, Functional Calculus of Pseudo-differential Boundary Prob̆lems, Birkauser, Boston, 1986. 
[LS] C. Lange and D. Smith, Singular Perturbation analysis of integral equations, Stud. Appl. Math. 79 no. 1 (1988), 1-63.

[MP] V. Mazja and B. Panejah, Degenerate elliptic pseudodifferential operators and the oblique derivative problem, Transactions of Moscow Math Society (1974).

[S] C. A. Shubin, Singularly perturbed integral equations, preprint.

[Sj] J. Sjöstrand, Operators of principal type with interior boundary conditions, Acta Math 130 (1973), 1-51.

[T] F. Treves, A new method of proof of the subelliptic estimates, Comm. Pure Appl. Math 24 (1971), 71-115.

[VG] M. Višik and V. Grusin, On a class of higher order degenerate elliptic equations, Math. USSR Sbornik 8 (1969), 1 - 32.

Department of Mathematics

California State University, Northridge

NORTHRIDGE, CA. 91330

AMS Classification: 45B11, 45E99, 34E15

Submitted: December 22, 1994

Revised: $\quad$ August 24, 1995 